\title{
Fast and Nondestructive Detection on the EVA Gel Content in Photovoltaic Modules by Optical Reflection
}

\author{
Heng-Yu Li, Yun Luo, Christophe Ballif, and Laure-Emmanuelle Perret-Aebi
}

\begin{abstract}
Poly(ethylene-co-vinyl acetate) (EVA) has been the dominating material in the photovoltaic (PV) encapsulant market for decades, owing to its superior cost-performance balance. To achieve its desired material properties, EVA undergoes a curing reaction during the module encapsulation process. The resulting EVA gel content after encapsulation is an important criterion for the module encapsulation quality control. Normally, the determination of gel content is achieved using a tedious solvent extraction method. In this paper, a fast and nondestructive detection method on the EVA gel content based on the optical reflection is explored. First, the homogeneity of the EVA gel content distribution after the standard EVA encapsulation process is studied. Then, the feasibility of the proposed optical approach applied to transparent modules is investigated. After that, a method is developed to apply it to opaque modules by incorporating a mirror into the module construction. It was found that the haze factor of the reflected light correlates well with the EVA gel content in the opaque modules. This proof-of-concept work could lead to the development of a fast and nondestructive tool for detecting the EVA gel content in both transparent and opaque PV modules, which is promising for integration as an inline diagnostic tool in the module manufacturing line.
\end{abstract}

Index Terms-Curing, optical reflection, packaging, photovoltaic cells, polymer films.

\section{INTRODUCTION}

$\mathbf{C}$ LEAN and renewable electricity resources are of utmost importance to the sustainable development of human society. As a promising candidate, photovoltaic (PV) technology and industry have both experienced tremendous growth over the past decades [1]. However, in order to further compete in utility scale with the traditional fossil fuel-based electricity generation, the levelized cost of electricity of PV technology still needs to decline further [2], [3]. Toward that aim, improvements in the reliability, i.e., the life time, of PV modules are critical.

The PV modules deployed in the natural environment are exposed to the combination of multiple stresses includ-

Manuscript received October 9, 2014; revised December 12, 2014 and January 12, 2015; accepted January 21, 2015. Date of publication February 16, 2015; date of current version April 17, 2015.

H.-Y. Li and C. Ballif are with the École Polytechnique Fédérale de Lausanne, Institute of Microengineering, Photovoltaics, and Thin Film Electronics Laboratory, 2000 Neuchâtel, Switzerland, and also with CSEM, PV-Center, 2000 Neuchâtel, Switzerland (e-mail: hengyu.li@csem.ch; christophe.ballif@epfl.ch).

Y. Luo is with PlusMat AG, 3054 Schüpfen, Switzerland (e-mail: Yun.luo@plusmat.ch).

L.-E. Perret-Aebi is with CSEM, PV-Center, 2000 Neuchâtel, Switzerland (e-mail: laure-emmanuelle.Perret@csem.ch).

Color versions of one or more of the figures in this paper are available online at http://ieeexplore.ieee.org.

Digital Object Identifier 10.1109/JPHOTOV.2015.2397603 ing UV radiation, heat, chemical ingress, mechanical impact, etc., [4]-[6]. To protect the active energy conversion unit, i.e., the solar cells from the harsh operational conditions, it is necessary to encapsulate them using a qualified packaging scheme. In fact, nearly all the failure modes discovered in the field-degraded modules are due to the failure of the module packaging [7], [8]. Thus, the encapsulation materials and the quality of the encapsulation process are decisive for the PV module reliability and, hence, the future of PV technology.

Typical crystalline Si-based PV modules are composed of a front glass superstrate, a glass or polymeric back substrate with the stringed solar cells are sandwiched in the middle [9]. To achieve structural integrity, two layers of encapsulants are utilized right above and below the cells. The encapsulant not only functions as the adhesive, but also as the optical index matching medium, as a barrier for chemical ingress (moisture, oxygen, etc.), as a heat conductor, and as a dielectric medium, etc. Currently, ethylene-co-vinyl acetate (EVA) occupies the majority of the PV encapsulant market. EVA encapsulant is normally crosslinked during the module encapsulation process in order to achieve the desired material properties. EVA reaches certain curing level at the end of the encapsulation process. In the $\mathrm{PV}$ industry, this is frequently represented by a value called gel content, which equals the weight percentage of the gel network (cross-linked portion) in the overall EVA.

Due to its importance, the EVA gel content is often adopted as criteria for judging the encapsulation quality of the PV modules. In the module manufacturing process, a number of modules have to be removed at a certain frequency for monitoring the EVA gel content inside. Historically, EVA gel content is measured by the solvent extraction method, where an organic solvent like toluene or xylene are used to dissolve the soluble portion and extract the cross-linked portion [10], [11]. The gel content is calculated as the weight percentage of the nonsoluble part of the EVA. Typically manufacturers require that the EVA gel content in the encapsulated modules should exceed $80 \%$. The solvent extraction method is not only time consuming (usually requiring about $24 \mathrm{~h}$ ), but a piece of EVA has to be taken from the PV module as well. It is destructive and implies an economic loss. In today's high-output PV module manufacturing lines, a much faster and nondestructive quality control method is in demand to ensure a high production yield.

More than ten types of different approaches have been proposed to develop fast quality control on the EVA gel content. Proposed techniques like Raman spectroscopy [11], scanning acoustic microscopy [12], differential scanning 


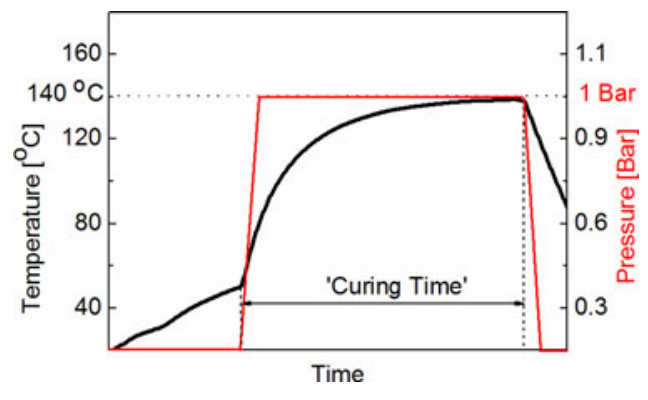

Fig. 1. Temperature and pressure profiles of the encapsulation process used in this study. The black line is the measured temperature in EVA throughout the process, with a heating plate temperature of $140{ }^{\circ} \mathrm{C}$. The red line represents the overall pressure applied to the laminates ( 1 bar during the curing process).

calorimetry (DSC) [13], [14], dynamic mechanical analysis (DMA) [15], [16], tensile test [11], etc. In [11], the performances of those proposed techniques have been compared and evaluated comprehensively. A few of them such as DSC and DMA remain to be destructive, although they can shorten time required per measurement. Previously, a fast and nondestructive approach for detecting the EVA gel content has been reported by our group, based on the UV/Vis spectroscopy [17]. The limitation of this development is that it relies on the optical transmission measurement and, hence, can only be applied onto transparent modules. Today, a majority of the PV modules in the market still use nontransparent polymeric backsheets as the substrate, which results in the opacity of the module. Here, this paper is intended to further develop the concept and allow it to work for both transparent and opaque modules. Such a technique will hold strong promise for use as an inline diagnostic tool for monitoring the EVA gel content in a PV module manufacturing line.

\section{EXPERIMENTAL SETUP}

\section{A. Encapsulation Process}

Various commercial grades of EVA encapsulants have been tested for the encapsulation, DSC, and optical studies. Due to the similarity of the results, only one representative EVA grade is presented here. The encapsulation and, thus, the curing of EVA are performed in a controlled manner in a flat-bed vacuum bag laminator (3S S1815 laminator). The temperature and pressure profiles of a typical lamination process are shown in Fig. 1. After a preheating step on pins for approximately $5 \mathrm{~min}$ (plate temperature at $140{ }^{\circ} \mathrm{C}$ unless otherwise mentioned), the pins are retracted downward and 1 bar pressure is immediately applied to the laminates. The pressure is applied starting at the onset of the curing time, which occurred under vacuum at below 1 mbar. After a preset curing time between 0 and $1300 \mathrm{~s}$, the pressure is removed and the sample is transferred out of the lamination chamber and cooled naturally in ambient environment [9].

\section{B. Differential Scanning Calorimetry Study}

EVA samples with different curing times are analyzed using a DSC setup (Mettler Toledo DSC1). The DSC samples are prepared as 1-mm thick circular discs of 5-10 $\mathrm{mg}$ in an aluminum crucible. Thermograms are recorded under constant nitrogen

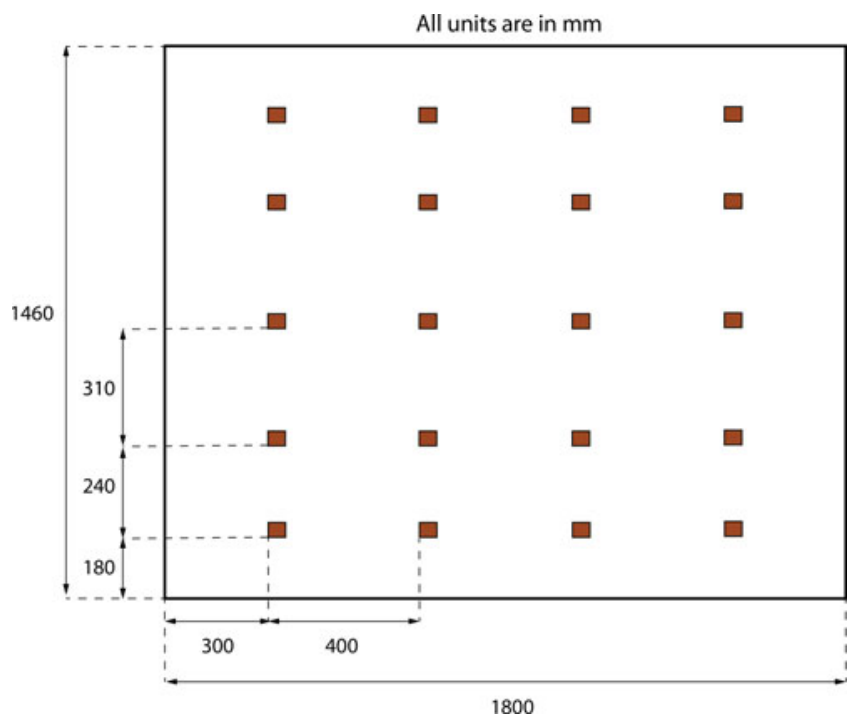

Fig. 2. Locations of temperature measurements on the heating plate. The number displayed is the dimension in millimeters.

flow from 30 to $200{ }^{\circ} \mathrm{C}$ at a heating rate of $15^{\circ} \mathrm{C} \cdot \mathrm{min}^{-1}$ (unless otherwise indicated).

\section{Measurement on the Temperature of Heating Plate}

The temperature measurement is performed with a PT100 T-sensor connected to a data logger. Measurements are performed on 20 locations on the heating plate. The distribution of the measurement sites are shown in Fig. 2, where the number displayed is the distance in millimeter. The size of the whole heating plate is $1800 \times 1460 \mathrm{~mm}^{2}$. The temperature of the heating plate is set to be $140{ }^{\circ} \mathrm{C}$ and dwell for $1 \mathrm{~h}$. At each measurement site, the T-sensor is pressed firmly onto the heating plate for $5 \mathrm{~min}$ till the temperature displayed in the data logger is stabilized, and then, the temperature is recorded.

\section{UV/VIS Spectroscopy}

The optical characterization on the encapsulants is carried out with a UV/Vis/NIR spectrophotometer (Perkin Elmer, Lambda 900). The measured wavelength range is set to be $320-2000 \mathrm{~nm}$. Total transmission and total reflection (TR) are obtained using an integrating sphere. From the UV/Vis spectroscopy, the haze factor of the transmitted or reflected light is calculated. The haze factor is defined as the percentage of the diffused light in the total transmitted or reflected light, which is often used to describe the extent to which light is diffused after traveling through a medium.

\section{RESULTS AND DisCUSSIONS}

\section{A. Evolution of Homogeneity of Ethylene-Co-Vinyl Acetate Gel Content Distribution}

Normally, the EVA gel content in the PV module is measured at one or a few spots in the module. To validate the representativeness of the measurement results, it is necessary to assess the homogeneity of the EVA curing level across the whole module. 


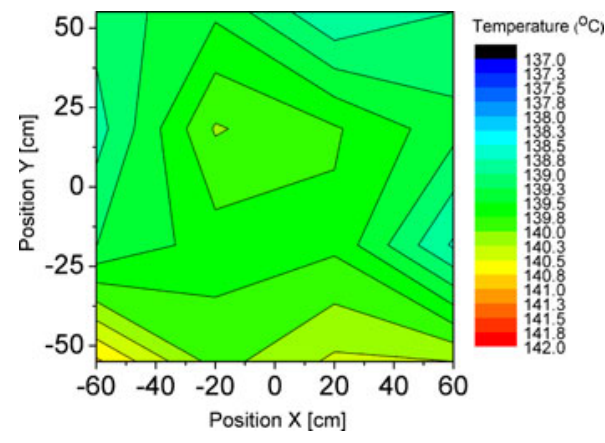

Fig. 3. Contour plot of the temperature distribution of the heating plate in the laminator.

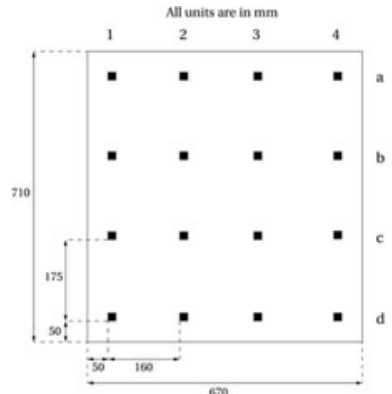

(a)

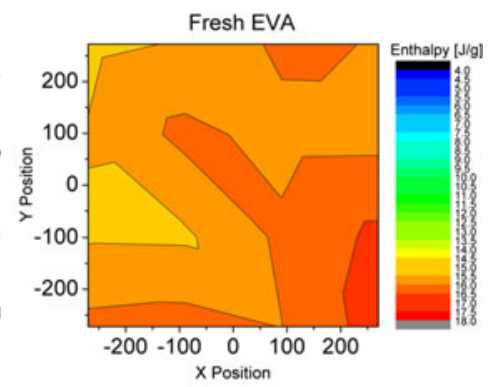

(b)
Fig. 4. Homogeneity of the residual additives distribution in the EVA encapsulant. (a) Locations of the EVA samples taken for the study of the additive distribution. (b) Contour plot of the enthalpy distribution in the uncured fresh EVA encapsulant.

As the curing of EVA is a thermally activated process following an Arrhenius-type temperature dependence, the temperature uniformity of the heating plate can greatly influence the homogeneity of the resulting EVA curing level. In this paper, the uniformity of the heating plate in the laminator is studied first.

The result of the temperature mapping is visualized in the contour plot in Fig. 3. As one can see, the heating plate exhibits a uniform temperature distribution with only a deviation of $\pm 1^{\circ} \mathrm{C}$. This result allows us to ignore the influence of the temperature uniformity of heating plate on the homogeneity of the EVA gel content distribution after the curing step.

Besides the temperature, the initial concentration of the curing agent (peroxides) in the uncured EVA encapsulant can also greatly affect the EVA conversion rate. Thus, it is equally important to know the homogeneity of initial additive (mainly peroxide) concentration distribution in the EVA. Here, DSC is used as the tool. As described in [13], the exothermic band in the DSC thermograph of the EVA encapsulant correlates well with the amount of residual peroxides in the EVA. For the DSC sample preparation, 16 samples are cut from 16 evenly spaced locations on a $670 \times 710 \mathrm{~mm}^{2}$ uncured fresh EVA encapsulant, as drawn in Fig. 4(a).

After the DSC measurements, the enthalpy of the exothermic band is integrated and used here to represent the concentration of the peroxides in the EVA sample. After having obtained the enthalpies of all 16 samples, a contour plot is generated to illustrate the homogeneity of the peroxide distribution in the uncured

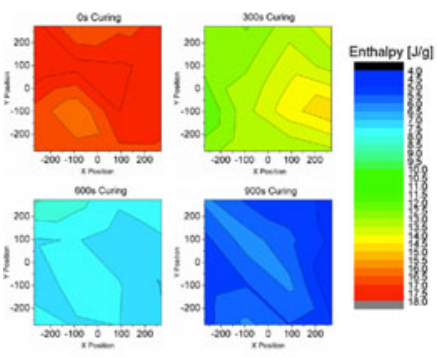

(a)

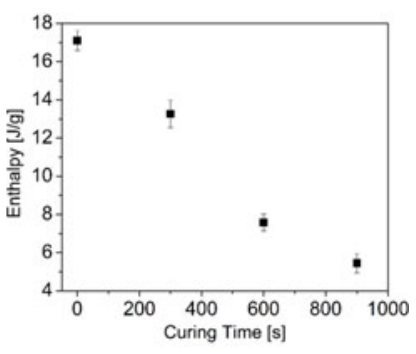

(b)
Fig. 5. Homogeneity of the residual additive distribution in the EVA encapsulant throughout the curing process. (a) Contour plot of the enthalpy distribution in EVA encapsulant after four different curing time: 0, 300, 600, and $900 \mathrm{~s}$. (b) Evolution of the enthalpy of the exothermic transition in the DSC thermogram of the cured EVA.

fresh EVA encapsulant. The result in Fig. 4(b) shows that the average enthalpy is $16.0 \pm 1.0 \mathrm{~J} / \mathrm{g}$. The small variation suggests that the initial peroxide distribution is rather homogeneous in this commercial-grade EVA encapsulant.

In the discussion above, the two major factors that could lead to the inhomogeneous distribution of the EVA gel content in the encapsulated module are analyzed, which are the uniformity of the plate temperature and the homogeneity of the initial peroxides distribution in the EVA encapsulant. During the module encapsulation process, other issues may also result in the inhomogeneity of the final EVA gel content, like uneven hot press throughout the module layup, glass warping during the preheating step, and location-dependent nonuniform presence of peroxides in EVA at elevated temperature, etc. Therefore, it is very useful to track the evolution of the homogeneity of the EVA gel content in the PV module throughout the encapsulation process.

For this study, four identical module layups are encapsulated with the same encapsulation process. Each of them is taken out at different stages of the process, namely $0 \mathrm{~s}$ cured (taken out immediately after preheating for $300 \mathrm{~s}$ ), $300 \mathrm{~s}$ cured (taken out at $300 \mathrm{~s}$ after the curing step starts), $600 \mathrm{~s}$ cured (taken out at $600 \mathrm{~s}$ after the curing step starts), and $900 \mathrm{~s}$ cured (taken out at $900 \mathrm{~s}$ after the curing step starts). For each module, 16 DSC samples are taken out in the same manner shown in Fig. 4(a). The encapsulation process of all the modules are as follows: $T_{\text {plate }}=150{ }^{\circ} \mathrm{C}$; preheating for $300 \mathrm{~s}$; hot press $=1$ bar. After the encapsulation, all modules are cooled down with uniform cooling across the module surface to eliminate the influence of the postcuring during cooling. Then, the DSC samples are cut out from the back side of the modules. The procedure of the DSC measurements follows the one for the fresh EVA reported above.

Fig. 5(a) shows the contour plots of the enthalpy distribution at all four stages of the curing steps. One can clearly see the good homogeneity and the development of the enthalpy in the encapsulation process. This development is due to the evolution of the peroxide consumption in the encapsulation process. Fig. 5(b) shows the enthalpies measured in the DSC plotted against the curing time. As the encapsulation progresses, the enthalpy measured in the EVA decreases, indicating the ascent of the EVA curing level. The error bars represent the standard deviation from the average enthalpy of the 16 samples. Notably, in the worst 


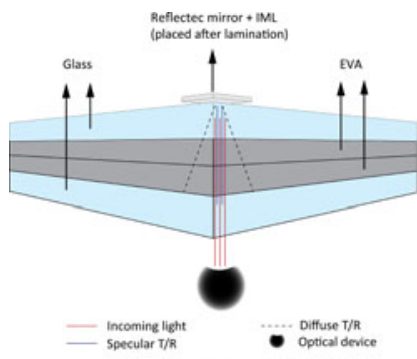

(a)

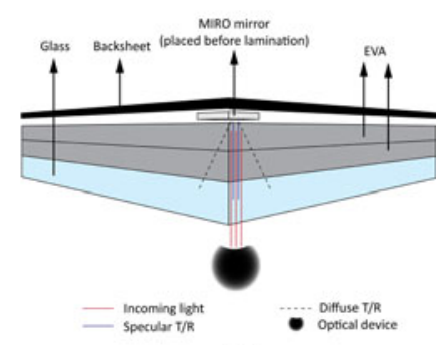

(b)

Fig. 6. Sketches of the sample configurations for the optical detection in reflection. (a) Transparent module with the mirror applied exteriorly after lamination. (b) Opaque module with the mirror embedded before lamination.

case, the standard deviation is less than $8 \%$ of the average value. Therefore, this leads to the conclusion that the distribution of the EVA curing level in the encapsulated PV module is sufficiently homogenous throughout the encapsulation process. It validates the representativeness of the results obtained by the technique to be developed here for EVA gel content detection in this paper.

\section{B. Optical Detection in Reflection Mode}

The optical detection in transmission mode on the EVA gel content in PV modules has been well documented in [17]. However, the optical detection in transmission mode only works for transparent PV modules. As the majority of crystalline PV modules currently use opaque backsheet instead of glass for the sake of lightweight, it limits greatly the application scale of our optical detection technique. Moreover, from the view point of the device development, it is also believed to be more convenient to operate in reflection mode than in transmission mode.

Here in this section, the development on the optical detection in reflection mode is presented. The front architecture of the PV module is designated to exhibit low reflection. Toward the development of the technique in reflection, first it is necessary to increase the reflection signal from the PV module. One idea is to use a mirror element to enhance the reflectivity. The paramount requirement on the material properties of this mirror material is the low scattering effect at the surface for the light of about $400 \mathrm{~nm}$. Ideally, the haze effect of the mirror itself should be much lower than the hazing effect induced by the EVA layer, in order to increase the sensitivity of this technique. The next requirement is the compatibility of the mirror material with the other module components and the encapsulation process. This will be addressed in the future work on the realization and commercialization of the actual device.

1) Optical Detection in Reflection Mode for Transparent Photovoltaic Modules: For transparent modules, the mirror needs not to be embedded into the module layup, as illustrated in Fig. 6(a). Instead, the mirror can be applied at the exterior of the module. Thus, the application of the mirror does not have to take place prior to the encapsulation process. For the transparent module, a mirror deposited on a plastic substrate (Reflectec) is selected for further test.

In Fig. 7, the TR and diffuse reflection (DR) measured on the Reflectec mirror are shown. At between 400 to $500 \mathrm{~nm}$,

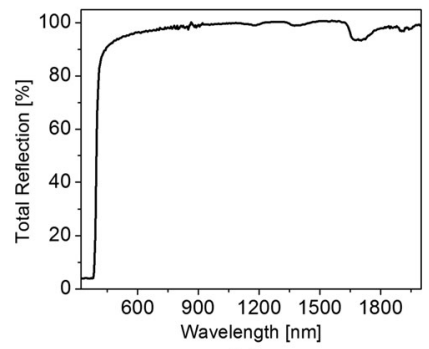

(a)

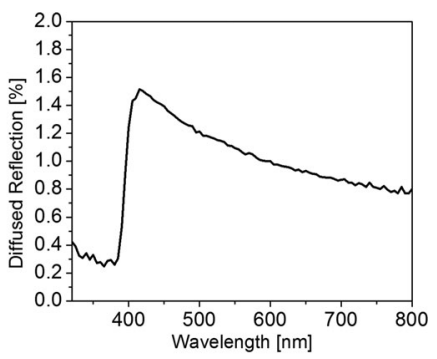

(b)
Fig. 7. (a) TR and (b) DR measured on the bare Reflectec mirror.

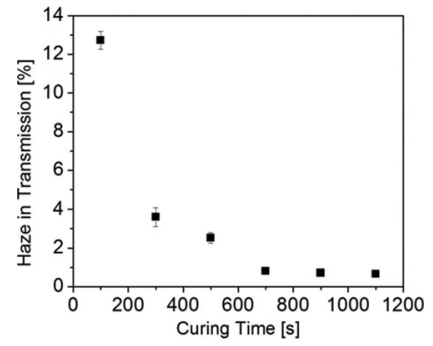

(a)

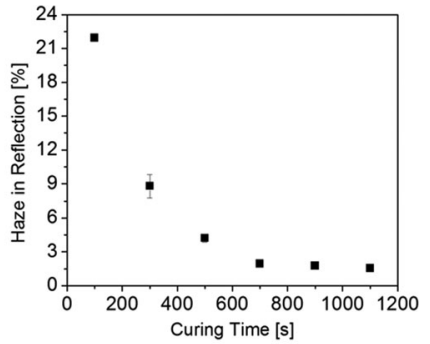

(b)
Fig. 8. Haze factor of the light leaving the glass-glass laminate with different curing time. (a) Haze factor of the transmitted light through the laminate. (b) Haze factor of the reflected light from the Reflectech mirror applied at the back of the laminate.

the TR of this mirror lies well above $80 \%$. The haze factor of the reflected light is lower than $2 \%$ in the studied wavelength range, with the maximum reached at about $400 \mathrm{~nm}$. The hazing effect fulfills the requirement. The main disadvantage of this mirror is its low working temperature at merely $60{ }^{\circ} \mathrm{C}$, which is considerably lower than the typical module encapsulation temperature. Experimentally, we have exposed this mirror to the actual encapsulation condition at $140{ }^{\circ} \mathrm{C}$. After the process, the mirror surface is severely deformed and forms numerous wrinkles, resulting in high hazing effect.

The procedure of the optical detection in reflection mode for transparent modules is proposed as follows. After the module encapsulation, the Reflectec mirror is applied onto the module surface, where no opaque objects like cells and metallizations present on top. To achieve better optical coupling, a layer of index matching liquid is used as the medium layer. The optical detection takes place on the other side of the module. To test this procedure, a series of minimodules are prepared under standard encapsulation process with the curing time of 100, 300, 500, 700, 900 , and $1100 \mathrm{~s}$. Total transmission and diffuse transmission are measured on these laminates. The haze factor of the transmitted light versus the EVA curing time is plotted in Fig. 8(a). The error bar represents the reproducibility of the data measured at five different locations on each sample. It is clearly seen that the haze factor of the transmitted beam correlates well with the EVA curing time.

Subsequently, the optical measurement in reflection is performed following the procedure described above. TR and DR are measured, and from which the haze factor of the reflected light is calculated. The results for different curing times are summarized in Fig. 8(b). Evidently, identical trend is observed 


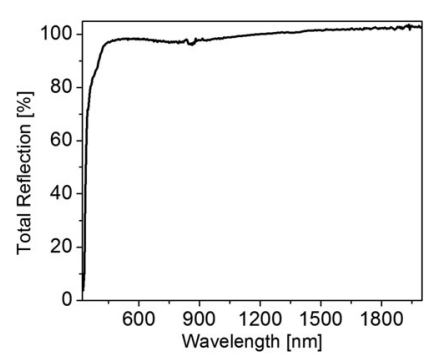

(a)

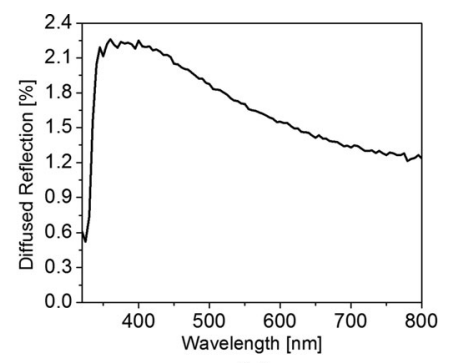

(b)
Fig. 9. (a) TR and (b) DR measured on the bare MIRO mirror.

here as in Fig. 8(a). By comparing both figures, noticeably, the detection in reflection mode results in similar sensitivity on the EVA gel content but with considerably larger absolute haze factors than those measured in transmission mode. This is partly due to the hazing effect of the Reflectech mirror, and is also related to the doubled optical path that the reflected light travels in EVA. To sum up, the concept of our optical approach in reflection mode is proved to work efficiently in transparent PV modules.

2) Optical Detection in Reflection Mode for Opaque Photovoltaic Modules: For opaque modules, it is proposed here to embed a mirror into the module layup, as shown in Fig. 6(b). The size of the mirror can be as small as $1 \times 1 \mathrm{~cm}^{2}$ in the present testing condition, which is the approximate size of the light spot in the UV/V is spectrometer. With the optimization on the device design, the area of this embedded mirror can be further reduced. In the case of opaque modules, the mirror will have to expose itself to the module encapsulation condition. The Reflectech mirror is not applicable due to its low working temperature. Instead, a type of aluminum mirror (4200 AG MIRO SILVER from Alanod) with a nominal thickness of $0.2 \mathrm{~mm}$ has been chosen for this study. The TR and DR of the MIRO mirror are first measured and shown in Fig. 9. Notably, MIRO mirror has reflection over $80 \%$ at $400 \mathrm{~nm}$, while the hazing effect is slightly higher than the Reflectech mirror.

To prove the proposed concept of the optical detection on EVA gel content in opaque modules, numerous glass/EVA/glass laminates are encapsulated for different curing time of 100,300, $500,700,900$, and $1100 \mathrm{~s}$. All of them are encapsulated with a piece of MIRO mirror. The mirror is located in between the EVA layer and the backsheet in this study. Moreover, to determine the actual EVA gel content after each encapsulation process, one additional EVA sample for DSC study is prepared in parallel.

Fig. 10(a) shows the evolution of the measured enthalpy with the EVA curing time. The small error bar shows the good reproducibility of these DSC measurements. The enthalpy is seen to decline with the increasing curing time. A correlation curve between the EVA gel content and the DSC enthalpy has been set up for the EVA used here [18]. This allows us to translate the enthalpy in Fig. 10(a) into the actual EVA gel content shown in Fig. 10(b). As expected, the EVA gel content increases as the EVA curing time is extended.

The TR and DR are measured at the embedded mirror in the modules with different curing time. From the results, the

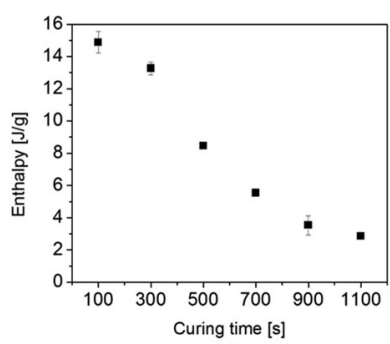

(a)

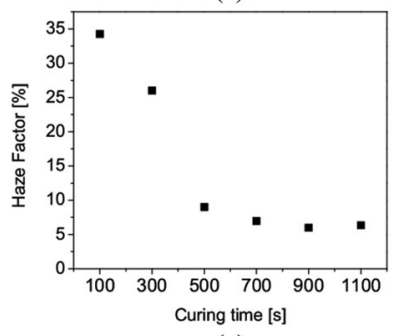

(c)

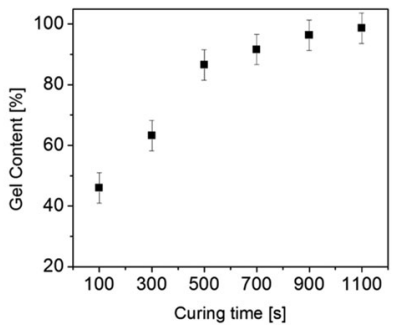

(b)

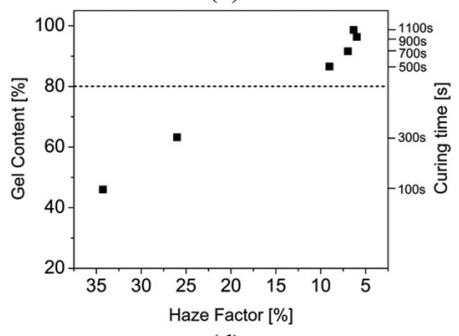

(d)
Fig. 10. Correlation between the EVA gel content and the haze factor of the reflected light from the MIRO mirror embedded into the opaque PV module. (a) Evolution of the enthalpy measured by DSC with the EVA curing time. (b) Development of the EVA gel content after different curing time. (c) Haze factor of the reflected light from the MIRO mirror embedded in between the EVA and backsheet. (d) Correlation between the haze factor of the reflected light and the EVA gel content. The dashed line at $80 \%$ represents the minimum EVA gel content typically required.

haze factors of the reflected light from the mirror at $400 \mathrm{~nm}$ are calculated and plotted in Fig. 10(c). As the curing time rises up, the haze factor of the reflected light through the EVA layer drops monotonically. Combining Fig. 10(b) and (c), the correlation between the EVA gel content and the measured haze factor can be constructed, as illustrated in Fig. 10(d). With the curing time longer than $500 \mathrm{~s}$, the resulting EVA gel content exceeds over $80 \%$. Fig. 10(d) shows that there exists a good correlation between the measured haze factor of the reflection from the module at the mirror site and the actual EVA gel content in the module. The measurement sensitivity of the optical detection is high, especially in the regime of low EVA gel content. There is certain difficulty in differentiating the EVA with gel content higher than $90 \%$. This is expected to be improved if an alternative mirror with even lower hazing effect is used. In the present module configuration, it is concluded that for the measured haze factors below $10 \%$ by the optical detection in reflection mode, the EVA gel content stays consistently above $80 \%$, as required by PV module manufacturers.

Currently, there is great interest from PV industries in the fast and nondestructive method for the determination of EVA gel content in PV modules. Several companies/institutes have also proposed other concepts based on different techniques [11], [19]. Compared to them, the advantages of the concept presented here are 1) high sensitivity in the range of high gel content (over $80 \%$ ), 2) potentially applicable to both glass/glass and glass/backsheet PV modules, and 3) ideal for inline diagnosis thanks to its nondestructive nature and unrivaled detection speed (time needed can be well below $5 \mathrm{~s}$ ). The potential problem of this concept is the embedding mirror needed in the case of the opaque modules, although its size can be minimized to be 
well below $1 \mathrm{~cm}^{2}$. In the following realization of the device based on this concept proved above, alternative solutions will be sought to either utilize existing module components (e.g., ribbons) as the mirror or to combine the embedded mirror with other functionalities in the future "smart" PV modules.

\section{CONCLUSION}

Nowadays, the PV industry highly desires a fast and nondestructive technique that can be integrated as an inline tool for detecting EVA gel content in PV modules. Toward this goal, an optical approach working in reflection mode based on the UV/V is spectroscopy is proposed here. The EVA gel content is first shown to be uniformly distributed in the studied module configuration after the standard EVA encapsulation process. Then, the proof of the proposed concept is achieved for both transparent and opaque PV modules. It is demonstrated that the proposed approach has high sensitivity and robustness on detecting the EVA gel content in transparent modules with no modification on the conventional module configuration. As for the opaque modules, a mirror is required to be integrated into the module configuration in this paper. A good correlation is found between the EVA gel content and the haze factor of the reflected light from the opaque module. The results show that the proposed approach can easily distinguish the EVA with gel content of below or above $80 \%$. This paper proves the feasibility of the proposed optical approach in reflection mode for detecting the EVA gel content in both transparent and opaque PV modules. It is expected that, with further optimization, it will become a strong candidate as the inline diagnostic tool for the gel content detection in the module manufacturing line.

\section{REFERENCES}

[1] IEA Int. Energy Agency. "PVPS report. A snapshot of global pv 1992 2012," Rep. IEA-PVPS T1-22, Paris, France, 2013.

[2] K. Branker, M. J. M. Pathak, and J. M. Pearce, "A review of solar photovoltaic levelized cost of electricity," Renew. Sustainable Energy Rev., vol. 15 , no. 9, pp. 4470-4482, 2011.

[3] S. B. Darling, F. You, T. Veselka, and A. Velosa, "Assumptions and the levelized cost of energy for photovoltaics," Energy Environ. Sci., vol. 4, no. 9 , pp. 3133-3139, 2011.

[4] A. W. Czanderna and F. J. Pern, "Encapsulation of pv modules using ethylene vinyl acetate copolymer as a pottant: A critical review," Solar Energy Mater. Solar Cells, vol. 43, no. 2, pp. 101-181, 1996.

[5] J. H. Wohlgemuth, M. Conway, and D. H. Meakin, "Reliability and performance testing of photovoltaic modules," in Proc. IEEE 28th Photovoltaic Spec., 2000, pp. 1483-1486.

[6] S. Kurtz, J. Wohlgemuth, T. Sample, M. Yamamichi, J. Amano, P. Hacke, M. Kempe, M. Kondo, T. Doi, K. Otani, "Ensuring quality of PV modules," in Proc. IEEE 37th Photovoltaic Spec. Conf. Ensuring Quality PV Modules, 2011, pp. 842-847

[7] C. Peike, P. Hülsmann, M. Blüml, P. Schmid, K.-A. Weiß, and M. Köhl, "Impact of permeation properties and backsheet-encapsulant interactions on the reliability of PV modules," ISRN Renew. Energy, vol. 2012, art. no. 459731-1-459731-5, 2012.

[8] S. Kurtz, J. Granata, and M. Quintana, "Photovoltaic-reliability R\&D toward a solar-powered world," Proc. SPIE, vol. 7412, pp. 74120Z-74120Z, Aug. 2009,

[9] R. F. M. Lange, Y. Luo, R. Polo, and J. Zahnd, "The lamination of (multi)crystalline and thin film based photovoltaic modules," Prog. Photovoltaics, Res. Appl., vol. 19, no. 2, pp. 127-133, 2011.

[10] Standard Test Methods for Determination of Gel Content and Swell Ratio of Crosslinked Ethylene Plastics. ASTM D2765-01, 2006.

[11] C. Hirschl, M. Biebl-Rydlo, M. DeBiasio, W. Mühleisen, L. Neumaier, W. Scherf, G. Oreski, G. Eder, B. Chernev, W. Schwab, and M. Kraft,
"Determining the degree of crosslinking of ethylene vinyl acetate photovoltaic module encapsulants-A comparative study," Solar Energy Mater. Solar Cells, vol. 116, pp. 203-218, 2013.

[12] W. Mühleisen, M. Biebl-Rydlo, M. Spielberger, "Determining the degree of EVA crosslinking in assembled PV modules acoustically and in-situ," in Proc. 26th Eur. Photovoltaic Sol. Energy Conf., 2011, pp. 3480-3483.

[13] H.-Y. Li, L. E. Perret-Aebi, R. Théron, C. Ballif, Y. Luo, and R. F. M. Lange, "Towards in-line determination of EVA gel content during PV modules lamination processes," in Proc. 25th Eur. Photovoltaic Sol. Energy Conf., 2010, vol. 515, pp. 4044-4046.

[14] M. Hidalgo, F. Medlege, M. Vite, C. Corfias-Zuccalli, P. Voarino, and J. González-León, "A new DSC method for the quality control of PV modules: Simple and quick determination of the degree of crosslinking of EVA encapsulants," Photovoltaics Int. J., vol. 14, pp. 130-140, 2011.

[15] M. Schubnell, "Investigation of the curing reaction of EVA by DSC and DMA," Photovoltaics Int. J., vol. 7, pp. 131-137, 2010.

[16] R. A. Mickiewicz, E. Cahill, and P. Wu, "Non-destructive determination of the degree of cross-linking of EVA solar module encapsulation using DMA shear measurements," in Proc. IEEE 38th Photovoltaic Spec. Conf., 2012, pp. 000710-000713.

[17] H.-Y. Li, L. E. Perret-Aebi, R. Théron, C. Ballif, Y. Luo, and R. F. M. Lange, "Optical transmission as a fast and non-destructive tool for determination of ethylene-co-vinyl acetate curing state in photovoltaic modules," Prog. Photovoltaics, Res. Appl., vol. 21, no. 2, pp. 187-194, 2013.

[18] Internal unpublished result.

[19] Laytec. Laytec x link for the evaluation of the level of eva cross-linking (2014) [Online]. Available: http://www.laytec.de/xlink/.

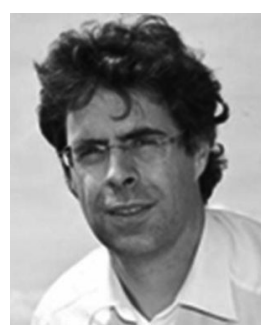

Christophe Ballif received the Master's degree in physics and the Ph.D. degree from École polytechnique fédérale de Lausanne (EPFL), Lausanne, Switzerland, in 1994 and 1998, respectively, focusing on novel photovoltaic materials.

He was a Postdoctoral Researcher with the National Renewable Energy Laboratory, Golden, CO, USA, where he was involved with compound semiconductor solar cells (CIGS and CdTe). He was then with the Fraunhofer Institute for Solar Energy Systems, Freiburg, Germany, where he was involved in mono- and multicrystalline silicon photovoltaics till 2003. He then joined the Swiss Federal Laboratories for Materials Testing and Research, Thun, Switzerland, before becoming a Full Professor and a Chair with the Institute of Microengineering, University of Neuchâtel, Neuchâtel, Switzerland, in 2004. In 2009, the Institute was transferred to EPFL. He is currently the Director of the Photovoltaics and Thin-Film Electronics Laboratory within the institute, as well as of the CSEM PV-Center. His research interests include thin-film solar cells, high-efficiency heterojunction crystalline cells, and module technology, contributing to technology transfer and industrialization of novel devices.

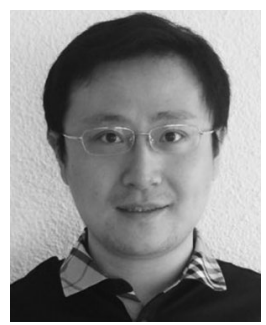

Heng-Yu Li received the B.E. degree in materials engineering from the Beijing University of Chemical Technology, Beijing, China, in 2004, the M.Sc. degree in nanosciences from the Delft University of Technology, Delft, The Netherlands, in 2006, and the Ph.D. degree in materials science from the Ecole Polytechnique Fédérale de Lausanne (EPFL), Lausanne, Switzerland, in 2013.

Since 2009, he has been working on the encapsulation process and the reliability of photovoltaic (PV) modules with the Photovoltaics and Thin-Film Electronics Laboratory, EPFL. In 2014, he joined the PV-Center, CSEM, Neuchâtel, Switzerland, where his focus is on the development of reliable speciality PV modules for various applications, including BIPV. 


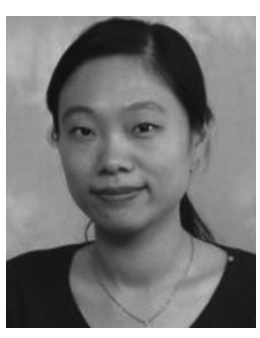

Yun Luo received the Ph.D. degree from the MaxPlanck-Institut für Mikrostruktur Physik, Halle, Germany, under the supervision of Prof. U. Gösele in 2005.

After three years as a Postdoctoral Fellow with the Ecole Polytechnique Fédérale de Lausanne, Switzerland, she joined Pasan SA as a Project Leader and continued as a Technology Manager with 3S Swiss Solar Systems AG and Meyer Burger Technology Group. In 2011, she founded Plus MAT AG, aiming to recycle Silicon from kerf loss for applications in photovoltaics, energy storage, and additive manufacturing.

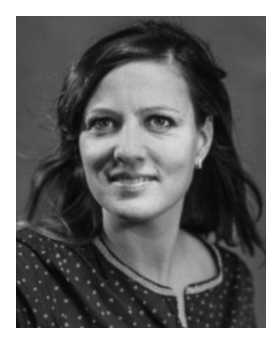

Laure-Emmanuelle Perret-Aebi received the Ph.D. degree in chemistry from the University of Fribourg, Fribourg, Switzerland, in 2004

After four years of Postdoctoral research with the University of Edinburgh, Edinburgh, U.K., and the University of Neuchâtel, Switzerland, in 2009 . she joined the PVlab of the Swiss Federal Institute of Technology (EPFL), Lausanne, Switzeralnd, as a Group Leader of PV module back-end activities. She is currently a Section Head with the "Modules \& Systems Integration" sector, CSEM PV-center, Neuchâtel, Switzerland. Her main research interest includes the development of innovative and highly reliable photovoltaic modules and systems for various applications, such as building, mobility, and land power plants. 